\title{
The study of different attention states under different background music based on Event-Related potential analysis
}

\author{
Yun Tang, Xin Deng ${ }^{\text {a }}$, Chenglin Zhang, Qiaosong Chen, Jin Wang and Qingyun Yang \\ Chongqing Key Laboratory of Computational Intelligence, Chongqing University of Posts and Telecommunications, \\ Chongqing 400065, China
}

\begin{abstract}
This paper conducts the research on the attention sates based on ERP analysis when the subjects are under the quiet, flute and zither background music. The amplitude and latency of P300 are analyzed. The results show that there are greater P300 amplitudes and smaller P300 latencies of $\mathrm{CZ}, \mathrm{PZ}, \mathrm{OZ}$ and $\mathrm{CP} 3$ in music background than those in quiet background. The PCA and ICA achieve to select the effective data components and the head model is reconstructed. The active degree of brain areas are analyzed by using the source location methods. The result shows that the brain's excitement is very obvious under the Bach's flute background. The study also indicates that some background music might help to improve the attention.
\end{abstract}

\section{Introduction}

Event-Related potential (ERP) and Functional Magnetic Resonance Imaging (fMRI) have been combined for using in the discussion of relationship between music and human cognition. When studying musical scale structures, Neuhaus [1] discovered that music and culture were closely related. In experiment, he found that the EEG signal containing a clear P300 component would present after the seventh deviant Thai tone, and P300 component with a longer latency could be brought when the eighth deviant Thai tone was given to the subjects. The results showed that not only Thai ton, but also other tones from different countries would induce a P300 component with larger amplitude and longer latency [1]. In addition, some researchers have showed that the cognitive processes on musical synchronization [2], music phase perception [3] and music scale structure [1] differed due to different cultures. However, some researchers have an opposite opinion that culture was not linked to music. They believed that the perception on music for people had the universality and dependence on the time [4], emotion [5], and feeling [6]. Morrison [7] used fMRI to compare the neural states of subjects when listening to familiar music from their native country and unfamiliar music from other countries. The result indicated that different cultural music might activate similar neurons [8]. Morrison hold the opinion that the subjects used the same way to comprehend the music, so they obtained the same understanding of its meaning.

We have briefly mentioned the attention, memory and observation above. Among them, the research on attention started several decades. Kerns [9] discovered that the early direct intervention could improve the attention of child with Attention Deficit Hyperactivity Disorder (ADHD). Noble [10] observed that the children in higher income family had the advantages of cognitive abilities

\footnotetext{
${ }^{\text {a }}$ Corresponding author :tangyunluck@163.com
} 
throughvarious neurocognitive systems. Kuniecki [11] proved that the red color was more capable to attract people's attention by the observation of EEG, especially in given context.

Graceful and soft music has the positive effect on psychology and physiology [12]. This paper discusses the relation between the music and the attention by analyzing the amplitude and latency of P300 component according to oddball paradigm. In our experiment, the brain active areas are located by using the source location methods and the active degree of each brain areas were analyzed. We obtain the result that the music might have a significant impact on the attention.

\section{Materials and methods}

\subsection{Subjects}

15 college students aged 20-30 years old are selected as the subjects randomly. They have equivalent educational background without a professional training about the music. Each subject is dextromanuality. Their intelligence mental and physical health are normal, and no one has mental disorders. All subjects volunteer to participate in this study.

\subsection{Background music selection}

A flute music and a zither music are chosen as the background music. The flute background music is the Bach's Sonata, and the zither background music is The Profound Mood between Clouds and Flowing. Another background is to keep quiet. In order to eliminate the physical differences, the time length of the selected music is consistent, so the different experimental data from the same subjects are produced from the different background music instead of other unrelated physical stimulation [13]. Thus, we set the power of the music around $100 \mathrm{~Hz}$ (Fig.1) in our experiment, which is presented to the subjects through the headphones.

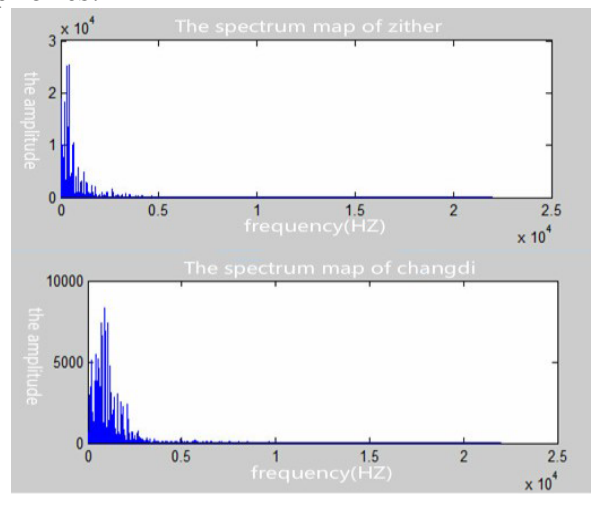

Figure 1. Spectrum map

\subsection{Experimental data acquisition}

The EEG recording device (Neuroscan) includes an electrode cap with 64 channels. The positions of electrodes are based on the international 10-20 system. The montage includes 8 midline sites (FPZ,FZ,FCZ,CZ,CPZ,PZ,POZ,OZ), 27 left hemisphere sites (FP1,AF3,F1,F3,F5,F7,FC1,FC3,FC5,FT7,C1,C3,C5,T7,CP1,CP3,CP5, TP7,P1,P3,P5,P7,PO3,PO5,P $\mathrm{O} 7, \mathrm{O} 1, \mathrm{CB} 1), \quad$ and 27 right hemisphere sites (FP2,AF4,F2,F4,F6,F8,FC2,FC4,FC6,FT8,C2,C4,C6,T8,CP2,CP4,CP6,TP8,P2,P4,P6,P8,PO4,PO6,P $\mathrm{O} 8, \mathrm{O} 2, \mathrm{CB} 2)$ [11]. To avoid the external disturbance, the tip of the nose is the reference for all electrodes and the blink response of the subjects is monitored by the Vertical-Electrooculogram 
(VEOG) signals and the Horizion-Electrooculogram (HEOG) signals. The resistance between the electrodes is less than $5 \mathrm{~K} \Omega$. The sampling rate is $1 \mathrm{KHz}$.

\subsection{Experimental process}

12 pictures are displayed to the subjects on a computer screen, including 4 words and 8 numbers (Fig. 2). The probability of numbers' appearance in this experiment is $80 \%$, and the probability of words' appearance is $20 \%$. The subjects need to click the left mouse button when watching the words. The pictures randomly flash every $125 \mathrm{~ms}$. The time of dark screen is $100 \mathrm{~ms}$ and one cycle is $800-1200 \mathrm{~ms}$.
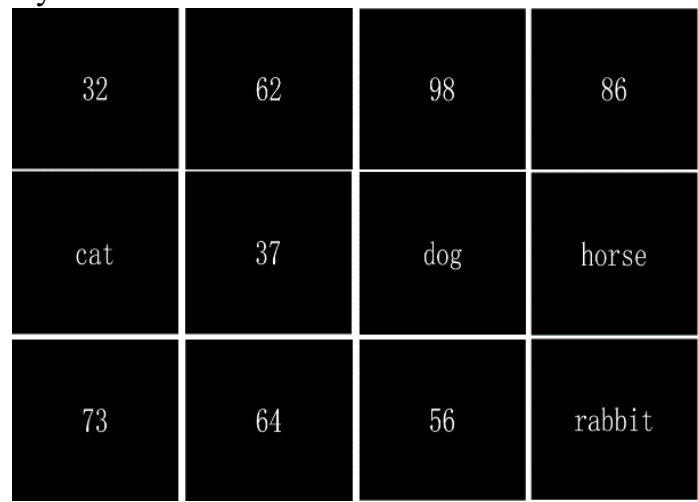

Figure 2. Pictures used for evoking the $\mathrm{P} 300$

\subsection{Data reprocessing}

In the experiment, the EEG signals are reprocessed including filtering and so on. Thus, a single experiment containing the ERP signal can be extracted. The process is as follows.

Baseline correction. Remove the Direct Current (DC) drift in the experiment.

Filtering. The signal of each channel is filtered by the Chebyshev band-pass filter with a frequency range of $0.1-30 \mathrm{~Hz}$. Then the power supply interference is removed by a notch filter for $50 \mathrm{~Hz}$.

Data adjustment. The blink or the rotation of the eyes, the slight movement of the subjects all can cause a larger amplitude of the EEG signals. However, the range of the normal amplitude of the EEG signal is between $-100 \mathrm{uV}$ to $100 \mathrm{uV}$. So the data whose amplitude are not in this range are eliminated to exclude the interference of the Electrocardiogram (ECG), the EOG, and the subjects' movement etc.

Single experimental data extraction. The single data segment of $700 \mathrm{~ms}$ are extracted from the whole data. The data starting from $100 \mathrm{~ms}$ before a single stimulus to $600 \mathrm{~ms}$ after the stimulus are overlaid to extract the ERP signal.

\subsection{ERP signal processed by PCA and ICA}

As the advanced neutral center of human, the brain depends on the mutual relation of different regions to control the body including all high-grade activities of human. The experiment chooses 64 electrodes recording EEG signals and $1 \mathrm{KHz}$ as the sampling rate. The dimensionality of the feature vector is $\mathrm{Ne} \times \mathrm{Nt}$, where $\mathrm{Ne}$ denotes the number of the electrodes and $\mathrm{Nt}$ denotes the number of the temporal samples in one trail. Thus, the large dimensions of EEG data are needed to remove the correlated data. The Principal Component Analysis (PCA) is used to descend the dimensions. Many original data with a certain correlation are recombined into a new set of irrelevant integrated data. In all linear combinations, the first principal component F1 is selected, which has the largest variance. If $\mathrm{F} 1$ is not enough to represent the original information of $t$ index, the second linear combination should be selected. Through the PCA algorithm, the unnecessary components are eliminated and the left can effectively reflect the information of the original signal. 
After the PCA processing, the data are consisted of a large number of unrelated source signals. The Independent Component Analysis (ICA) is a multi-channel signal decomposition method and derived from blind source separation. It is used to eliminate the unnecessary components within the time ranges of the analysis. Through the ICA, the data are separated and the independent components are obtained. In all of the processing, the effective components of the SNR are higher than the noise level, and the left components have a major impact on the Mean Global Field Power (MGFP).

The PCA and ICA are used to eliminate the unnecessary components, so the number of data are decreased. Finally, the source location methods including the moving dipole localization method and the current density method are applied at the independent EEG data.

\subsection{Head models reconstructing}

A realistic head model based on each subjects' anatomical information is created, which can increase the accuracy of source location. Based on the measured EEG, we also obtain the sensor positions, the head model, and a fit of one dipoles. For each fitted dipole, a confidence ellipsoid is computed both in the time domain and the frequency domain, which visualizes the localization accuracy.

After the head model is reconstructed, possible sites of excitation in different brain regions are tracked by using moving dipole localization method. Meanwhile, the excitation degree of each brain region is analyzed by using the current density method.

\section{Result analysis}

\subsection{ERP data analysis}

As CZ, PZ, OZ located in the sagittal line [14] and CP3 located in the auditory central region [15], these 4 electrodes are chosen to be observed in this experiment. The ERP is the averaged evoked potentials, which contains some components closely related to the psychological research, such as P300 and MMN (Mismatch Negativity). The P300 component is analyzed in this experience, which can be clearly noticed (Fig.3, 4, 5). The positive peak is appeared around $481 \mathrm{~ms}$ in the quiet background (Fig.3), around $469 \mathrm{~ms}$ in the flute background (Fig.4), and around $464 \mathrm{~ms}$ in the zither background (Fig.5). In order to analyze the influence of different background music on the ERP, the ERP data are analyzed statistically.

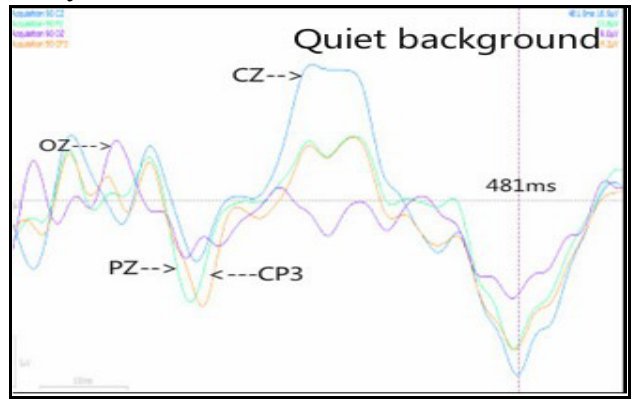

Figure 3. The ERP in quiet background

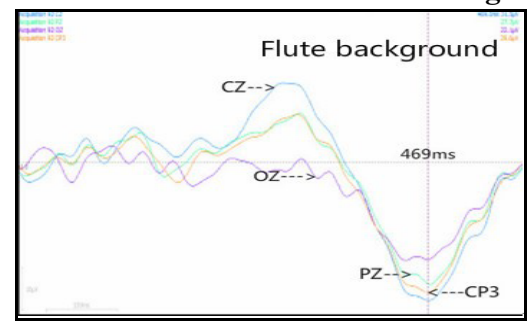

Figure 4. The ERP in flute background

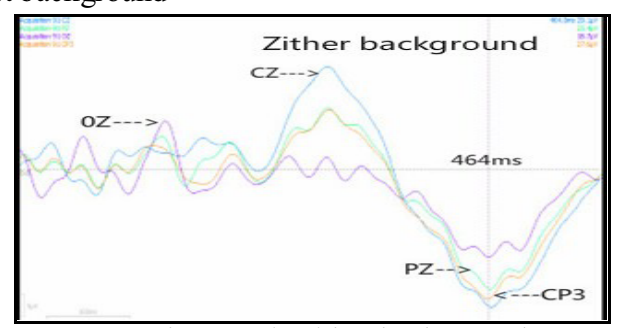

Figure 5. The ERP in zither background 


\subsection{Analysis of the latency and amplitude of P300}

In different background music, the latency of $\mathrm{P} 300$ for $\mathrm{CZ}$ and $\mathrm{PZ}$ varies a little, and the difference is within 10ms (Fig. 6). The latencies of P300 for OZ and CP3 vary a lot. For OZ and CP3, the latencies are longest in a quiet background, and are shortest in the zither background music. These results indicate that music might have a certain effect on the latency of P300. The shorter latency in the flute and zither background music showed that the subjects might have a better ability to response in this environment. In conclusion, the background music might have an influence on people's attention [16], and the zither background music might have a better effect than the flute background music. Fig.7 also illustrates these conclusions.

In the flute background, the amplitudes of $\mathrm{P} 300$ of $\mathrm{CZ}, \mathrm{PZ}, \mathrm{OZ}, \mathrm{CP} 3$ are bigger than those in the zither background music, and both of them are higher than those in the quiet background (Fig. 8). The amplitudes of P300 reflect the psychological involvement level of subjects [16]. Thus, the subjects' attention may differ in different background music. In summary, the attention of subjects might be improved in zither and flute background. Fig.9 also illustrates these conclusions.

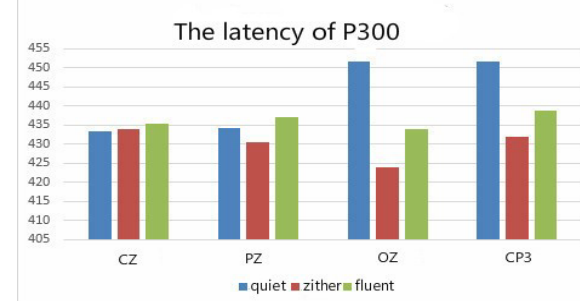

Figure 6. The latency of $\mathrm{P} 300$

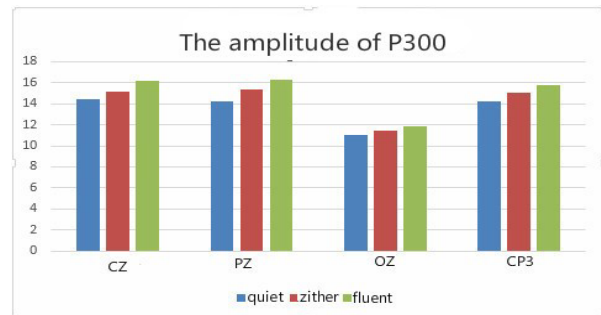

Figure 8. The amplitude of $\mathrm{P} 300$

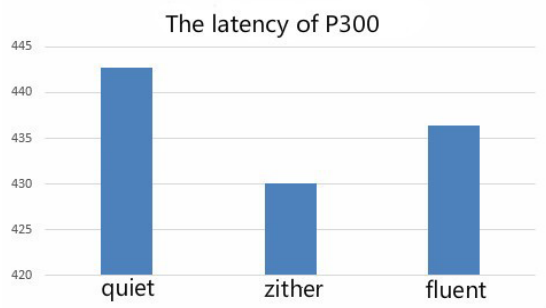

Figure 7. The latency of $\mathrm{P} 300$ statically

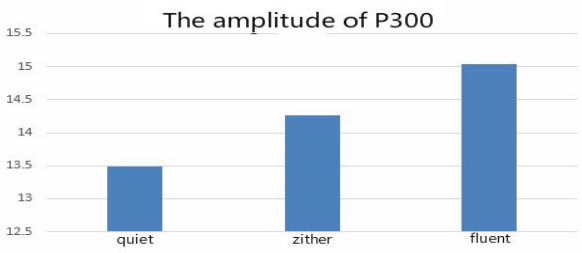

Figure 9. The amplitude of P300 statically

\subsection{Spectrum analysis}

The EEG signals can be divided into $\delta$ wave (Delta), $\theta$ wave (Theta), $\alpha$ wave (Alpha), $\beta$ wave (Beta) and $\gamma$ wave (Gamma). These five waves can reflect the human's consciousness and the high level of mental activities. The $\delta$ wave is relevant to the information fusion, the attention and the logic language information processing in cortical layer. The $\theta$ wave is concerned with a variety of cognitive functions. The $\alpha$ wave is related to the activity in cortical layer, especially the attention and the memory. The $\beta$ wave is interrelated with the attention, which can improve the cognitive abilities. The $\gamma$ wave emphasizes the integration of visual attention and the synchronization information of the brain rhythm [17].

The amplitudes of different waves in different backgrounds are shown in the table1. Except the four waves, other spectral amplitudes are too small to analyze. Obviously, in the three backgrounds, the changes mostly take place among the $\delta$ wave, the $\theta$ wave and the $\alpha$ wave. The $\delta$ wave and the $\theta$ wave, commonly known as "sleepy wave" [19], can reflect the arousal level [18] to some extent, and often disappears in the sleep and the trance state. Usually, the $\delta$ wave emerges in the deep sleep, and the $\theta$ wave appears in the shallow sleep. At this time, the subject's body is in a relaxed state and has a conscious awareness of alertness when the $\theta$ wave is produced. In our experiment, the amplitude of the $\delta$ in the quiet background is bigger than those in other two background music. The amplitude of $\theta$ 
wave in the quiet background is slightly smaller than those in other two background music. Compared with the $\theta$ wave, the $\delta$ wave is bigger. Therefore, the $\theta$ wave can be regarded as a quantitative, which means that the minor differences of $\theta$ might be caused by the psychological cues in different backgrounds. The result shows that subjects are more likely to be sleepy and fall into a deep sleep in a quiet background. Meanwhile, the subjects are easily fall into a light sleep and their alertness increases slightly in the zither and flute background. Besides, the greater amplitude of $\alpha$ wave, which reflects the relaxed state, shows that the subjects are more relaxed in the zither and flute background.

Table 1. The amplitudes of different waves in different backgrounds

\begin{tabular}{|c|c|c|c|c|}
\hline wave $(\mathrm{uV})$ & $\delta$ & $\theta$ & $\alpha$ & $\beta$ \\
\hline quiet & 8.0 & 0.5 & 0.4 & 0.3 \\
\hline zither music & 6.0 & 0.8 & 0.6 & 0.3 \\
\hline flute music & 5.0 & 0.8 & 0.6 & 0.3 \\
\hline
\end{tabular}

\subsection{The source location}

The source location methods include the dipole localization method and the current density reconstruction method. The position, direction and intensity of the dipoles are observed by the dipole localization method. With the position, direction and intensity information, we can obtain the active brain areas and track the variety of EEG signals. Then the current density reconstruction method is used to gain the current density of each brain and to analyze the excitation degree. There three factors are used to describe the result, which are background music, brain sites, and lateralization.

In the quiet background, the excitement appears on both sides of the auditory central region, and obviously the left excitation degree is greater than that on the right side (Fig.10). This result might be caused by two reasons. Firstly, the subjects who are dextromanuality have an advantage in the left of the brain. Secondly, the stereo music could make the listening asymmetry. In the zither background, the excitement only emerges on the right side of the auditory center (Fig.11). In the flute background, the excitement strongly occurs in the whole back of the head (Fig.12). Compared with these two result, the flute background brings the bigger excitation degree than those in zither background. Through the investigation of the subjects, the reason may be that the subjects are more familiar with flute. The result shows that the different backgrounds evoke the different excitement degree of brain areas.

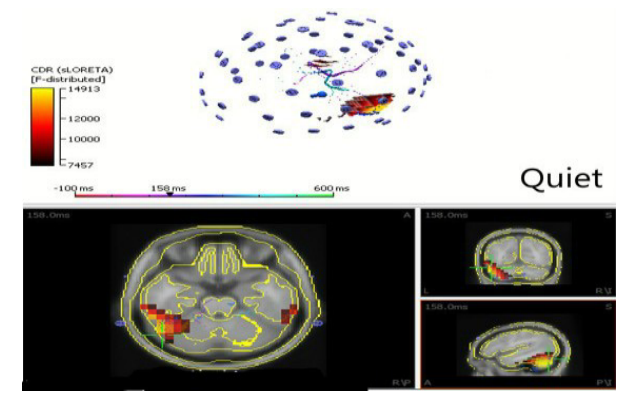

Figure 10. The excitement degree in quiet 


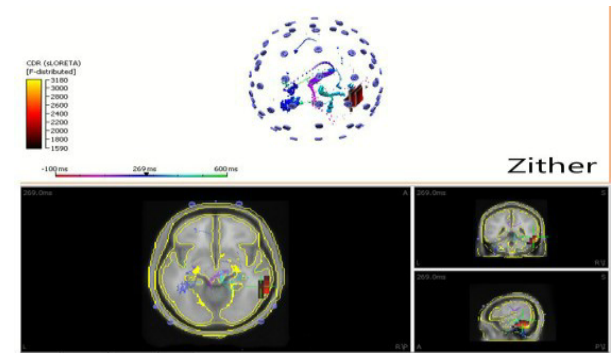

Figure 11. The excitement degree in zither

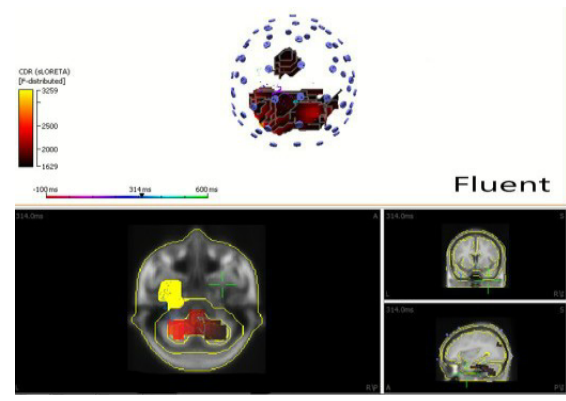

Figure 12. The excitement degree in flute

\section{Discuss}

The data and results of this experiment are analysed and summarized as follows.

Latency: The latencies of P300 are different in three different background music. However, the differences among them are small. Usually, the latency can reflect the reaction rate of subjects. But the small differences cannot prove that the three different background music would yield the different reaction rate. Here we conclude that the reaction rate is mainly depended on the individual difference and does not have the relationship with the environment.

Amplitude: In the flute background, the amplitude of P300 is bigger than those in the zither background music, and both of them are higher than those in the quiet background. We explain this result as the interference of the sound background. Compared with the quiet background, the subjects are more relaxed, and have a more concentrated attention in the zither and the flute background music. Meanwhile, the spectrum analysis equivalently illustrates the result. In the quiet background, there are bigger $\delta$ wave (value $=8 \mathrm{uV}$ ), smaller $\alpha$ wave $($ value $=0.4 \mathrm{uV}$ ) and $\beta$ wave (value $=0.3 \mathrm{uV}$ ) than those in other backgrounds. The result shows that the subjects are more likely to be sleepy and fall into a deep sleep in the quiet background. Contrarily, in the flute and zither background, the subjects are easy to fall into a light sleep and their alertness increase slightly. At the same time, they are more relaxed or easy to relieve tension.

Source location: It appears that the largest brain areas involved and the strongest excitement degree of brain are in the flute background. They are explained as following. Every object has its own natural vibration frequency, the same as every brain area of human. The music waves utilize the resonance with some brain regions, which can yield the relevant brain areas more active. It is noteworthy that Bach's sonata is selected as the flute background. According to the relevant research, Bach's music is more likely to resonate with the human organs.

In summary, the study indicates that some background music might help to improve the attention.

In this work, the subjects' EEG signals are recorded in three backgrounds. We have shown that the certain background music may help to improve the subjects' attention. However, there are some limitations in this work. One is the number of the subjects is limited. The other is that the recording time is not long enough. Thus, the future improvements to the work presented here might be consisted of two parts. One is to prolong the time of experiment, such as 45 minutes, to verify whether the conclusion of this paper works well. The other is to increase the types of music, such as jazz, Waltz, etc.

\section{Acknowledgment}

This work was partially supported by National Natural Science Foundation of China (No.61403054), the Fundamental and Frontier Research Project of Chongqing under Grants (No.cstc2014jcyjA40001, No.cstc2014jcyjA40022) and the Science Foundation Project of CQ Education Commission under Grant (No. KJ1400436). 


\section{References}

1. Neuhaus C. Perceiving musical scale structure: A cross-cultural event-related brain potentials study. Ann N Y Acad Sci, 999, 184-188(2003).

2. Drake C, Ben EI Heni J. Synchronizing with music: intercultural differences. Ann N Y Acad Sci, 999, 429-437(2003).

3. Nan Y, Knösche TR, Friederici AD. The perception of musical phrase structure: a cross-culturals study. Brain Res. 1094, 179-19(2006).

4. Drake C, Bertrand D. The quest for universals in temporal processing in music. Ann N Y Acad Sci, 930, 17-27(2001).

5. Balkwill LL, Thompson WF. A cross-cultural investigation of the perception of emotion in music: psychophysical and cultural cues. Music Percept, 17, 43-64(1999).

6. Smith LD, Williams RN. Children's artistic responses to musical intervals. Am J Psychol, 112, 383-410(1999).

7. Morrison SJ, Demorest SM, Aylward EH, Cramer SC, Maravilla KR. FMRI investigation of cross-cultural music comprehension. Neuroimage, 20, 378-384(2003).

8. Demorest SM, Morrison SJ. Exploring the influence of cultural familiarity and expertise on neurological responses to music. Ann N Y Acad Sci, 999, 112-117(2003).

9. Kimberly A. Kerns, Karen Eso, Jennifer Thomson. Investigation of a Direct Intervention for Improving Attention in Young Children with ADHD. Developmental Neuropsychology. 16(16), 273-295(1999).

10. KG Noble, MF Norman, MJ Farah. Neurocognitive correlates of socioeconomic status in kindergarten children. Developmental Science, 8(1), 74-87(2005).

11. M Kuniecki, J Pilarczyk, S Wichary. The color red attracts attention in an emotional context. An ERP study. Frontiers in Human Neuroscience, 9,212(2015).

12. Hwang, R. H. \& Shih, Y. N. Effects of background music on concentration of workers. WORK: A Journal of Prevention, Assessment, \& Rehabilitation, 38(4), 383-387(2011).

13. HaiWei Liu. Brain Research of Music Cognition and EEG Computational Analysis [D]. Xiamen University, 13-23 (2008).

14. Wei-Na ZHU, Jun-Jun ZHANG. Differential cognitive responses to guqin music and piano music in Chinese subjects: an event-related potential study. Neurosci Bull, 24(1), 21-28 (2008).

15. WeiCheng Lin. Research about Pattern Classification of Recognition Tasks Based on EEG Signals [D]. Hangzhou Dianzi University, 9-20 (2013).

16. Yu Ji. Research of P300 Processing Algorithm Based on Independent Component Analysis [D]. Zhejiang University, 10-14 (2013).

17. QiuJing Xu. Research on Visual Attention Classification Based on EEG Entropy Parameters [D]. Tianjin University University, 4-6 (2009).

18. Tran Y, Craig A, Boord $\mathrm{P}$, et al. Personality trails and its association with resting regional brain activity[J]. International Journal of Psychophysiology, 60(3), 215-224 (2006).

19. MaoMao. Psychologically and Physiologically Measuring Musichinduced Emotion and its Application in Service Design [D]. Tsinghua University, 11-18 (2013). 